\title{
Análisis de la actividad sexual de gestantes sin riesgo obstétrico que acuden a Hospitales públicos de Lima Metropolitana, Perú
}

\author{
Alex Guibovich Mesinas ${ }^{1 a}$, Tomás Angulo Delgado ${ }^{1 a}$, Elmer Luján-Carpio ${ }^{2 b}$
}

\section{RESUMEN}

Objetivo: conocer las características de la actividad sexual en gestantes sin riesgo obstétrico que acuden a hospitales públicos de Lima Metropolitana.

Material y Métodos: Estudio exploratorio, multicéntrico, descriptivo y transversal, realizado durante el primer semestre del 2014 en 9 hospitales públicos de Lima Metropolitana. La muestra fue conformada por las gestantes sin riesgo obstétrico que asistieron a los Consultorios Externos de los Servicios de Obstetricia. Se usó un modelo no probabilístico por conveniencia. En quiénes aceptaron voluntariamente participar y firmaron el consentimiento informado, se realizó una encuesta validada por expertos. La información fue analizada usando el software STATA 21.

Resultados: Se encuestaron 1991 gestantes, 924 (46,4\%) tenían entre 15 y 25 años, la mayoría eran casadas, y católicas $(81,8 \%$ y $1380(69,3 \%)$ habían nacido en Lima. La mayoría, era multigesta $(46,3 \%)$, con embarazo a término y $86,3 \%$, afirmó no haber tenido RN pre-término. $1347(67,6 \%)$ tenían parto vaginal. La frecuencia de actos sexuales, el deseo sexual, el patrón de respuesta y el miedo a ejercer relaciones sexuales disminuyó de periodicidad a mayor edad gestacional. El miedo a tener actos sexuales aumentó de 12,6\% a 76,2\% en el tercer trimestre. La mayoría, refirió ausencia de orgasmo durante el coito $(42,8 \%)$. La posición para el acto sexual pasó de "Él sobre ella" durante el primer trimestre a "De lado", durante el tercer trimestre, con significancia estadística.

Conclusión: A mayor edad gestacional, la actividad sexual en las gestantes de Lima Metropolitana tiende a disminuir en frecuencia, deseo, y ciclo de respuesta. Siendo la posición "De lado" la más utilizada en el tercer trimestre.

(Horiz Med 2015; 15(3): 6-12)

Palabras clave: sexualidad, embarazo, actividad sexual, pareja sexual. (Fuente: DeCS BIREME).

\section{Analysis of sexual activity in pregnant women without obstetric risk attending public hospitals in Lima, Peru}

\begin{abstract}
Objective: to know the characteristics of sexual activity in pregnant women without obstetric risk who attend public hospitals in Lima.

Material and Methods: exploratory, multicenter, descriptive and cross-sectional study, conducted during the first half of 2014 in 9 public hospitals in Lima. The sample was made up of pregnant women without obstetric risk who attended the outpatient clinic of obstetric departments. A non-probabilistic model is used for convenience. An expert validated survey was conducted on patients who voluntarily agreed to participate and signed the informed consent. The information was analyzed using STATA 21 software.

Results: 1991 pregnant women answered the survey, 924 (46.4\%) were between 15 and 25 years, most were married, and Catholic (81.8\%) and 1380 (69.3\%) were born in Lima. Most had several previous pregnancies (46.3\%), with term pregnancy and $86.3 \%$ informed they had no pre-term infants. 1347 (67.6\%) had vaginal delivery. The frequency of sexual acts, sexual desire, the response pattern and the fear of exercising sexual intercourse decreased at higher gestational age. Fear of sex acts increased from $12.6 \%$ to $76.2 \%$ in the third trimester. Most informed of a lack of orgasm during intercourse $(42.8 \%)$. The position for intercourse went from "him on her" in the first trimester to "sideways" during the third trimester, with statistical significance.

Conclusion: sexual activity in pregnant women of Metropolitan Lima tend to decrease in frequency, desire, and response cycle. The "sideways" position was the most often used in the third trimester. (Horiz Med 2015; 15(3): 6-12)
\end{abstract}

Key words: sexuality, pregnancy, sexual activity, sexual partner. (Source: MeSH NLM).

Ginecólogo - Obstetra. Hospital Nacional Arzobispo Loayza. Lima-Perú

Profesor de Medicina de la Universidad de San Martín de Porres. Lima-Perú

Facultad de Medicina Humana de la Universidad de San Martín de Porres. Lima-Perú

Sociedad Científica de Estudiantes de Medicina de la Universidad de San Martín de Porres. Lima-Perú 


\section{INTRODUCCIÓN}

La gestación, conlleva en la mujer a tener una serie de cambios: psicológicos, sociales y biológicos; teniendo como eje, su antecedente cultural, que define significativamente la conducta en varios ámbitos como en los cuidados prenatales, salud, alimentación, estrés y relaciones sexuales (1-3).

La conducta sexual durante el embarazo constituye un aspecto importante dentro de la vida de la mujer y es determinante para fortalecer la unión con su pareja; en nuestro país, así como en otros de Latinoamérica, la sexualidad todavía se encuentra rodeada de prejuicios, generalmente por la falta de una adecuada educación sexual.

Una buena relación sexual durante el embarazo, es determinante para fortalecer la unión de la pareja, además; ambos deben asumir nuevos roles: el de padres, sin dejar de ser amantes(4).

Existen diversos estudios que exploran la sexualidad durante la gestación(3-8), muchos de estos estudios han sido realizados hace décadas y tuvieron como limitación metodológica el tamaño de la muestra(9), esto es importante de remarcar ya que las conductas sexuales cambian culturalmente con el paso de los años(10) y el tamaño de la muestra refleja la validez interna del estudio $(11,12)$.

Tenemos evidencia de distintas partes del mundo, incluyendo: Europa, Asia y América; sin embargo, en el caso de América Latina, los estudios son mínimos y, siendo más específicos; en el caso del Perú, los estudios han sido dirigidos para evaluar a las gestantes adolescentes (13).

Conocer más acerca de la sexualidad humana es muy importante para mejorar el bienestar y la salud de los individuos, puesto que, la ignorancia y nociones erróneas sobre la actividad sexual, tienen estrecha relación con los diversos problemas de salud y con la calidad de vida, especialmente durante la gestación, proceso en el que podrían asociarse factores de riesgo y conducta inadecuada por desinformación o desconocimiento.

El objetivo de este estudio, fue conocer las características de la actividad sexual en gestantessin riesgo obstétrico que acudían a su control prenatal en hospitales públicos de Lima Metropolitana.

\section{MATERIAL Y MÉTODOS}

Tipo de estudio, temporalidad y espacio:

Estudio exploratorio, multicéntrico, descriptivo y transversal, realizado durante el primer semestre del 2014 en 9 hospitales públicos de Lima Metropolitana: Hipólito Unanue, Edgardo Rebagliati, Daniel Alcides Carrión, San Juan de Lurigancho, Arzobispo Loayza, Carlos Lanfranco La Hoz, Guillermo Almenara Irigoyen, Instituto Nacional Materno Perinatal y Dos de Mayo.

\section{Muestra y muestreo:}

La muestra fue conformada por 1991 gestantes sin riesgo obstétrico que asistieron a los Consultorios Externos de los Servicios de Obstetricia de distintos hospitales nacionales de Lima Metropolitana, Perú; la elección de la muestra siguió un modelo no probabilístico por conveniencia.

\section{Método de recolección de datos:}

La gestante que aceptó voluntariamente participar, brindó anónimamente sus datos al investigador, quién completo la encuesta. Se obtuvo el consentimiento informado de la gestante y de su progenitor, cuando era menor de edad y se procedió con las preguntas estructuradas.

Se aplicó una encuesta Ad hoc, sus preguntas evaluaron 4 periodos: el periodo antes del embarazo y los tres trimestres de gestación; además, las preguntas abordaron las características sociodemográficas, gravidez, paridad, práctica y frecuencia de relaciones sexuales, patrón del ciclo a respuesta sexual, miedo a ejercer relaciones sexuales y posiciones sexuales practicadas por las gestantes.

El instrumento utilizado fue brindado por la Cátedra de Ginecología y Obstetricia de la Facultad de Medicina Humana de la Universidad de San Martín de Porres, a cargo del Doctor Napoleón Paredes Pérez y validado por todos los Coordinadores de la Asignatura en las Sedes Hospitalarias.

\section{Análisis estadístico:}

La información fue analizada usando el software STATA 21. Se hizo la comparación entre trimestres.

Las variables fueron en su totalidad cualitativas, por eso los resultados fueron expresados en tablas descriptivas de frecuencia y porcentajes. 


\section{RESULTADOS}

Se encuestaron 1991 gestantes. La tabla 1, resume las características demográficas de las gestantes estudiadas. Del total, $924(46,4 \%)$ gestantes tenían una edad entre el rango de 15 y 25 años, el $49,6 \%$ de las gestantes eran casadas, la religión mayoritaria fue la católica con $1628(81,8 \%)$ y 1380 (69,3\%) gestantes habían nacido en Lima.

Tabla 1. Características demográficas de las gestantes

\begin{tabular}{|c|c|c|}
\hline & $\mathbf{N}$ & $\%$ \\
\hline \multicolumn{3}{|l|}{ Edad } \\
\hline $15-25$ & 924 & 46.4 \\
\hline $26-35$ & 913 & 45.9 \\
\hline$>35$ & 154 & 7.7 \\
\hline \multicolumn{3}{|l|}{ Estado Civil } \\
\hline Soltera & 589 & 29.6 \\
\hline Casada & 988 & 49.6 \\
\hline Conviviente & 414 & 20.8 \\
\hline \multicolumn{3}{|l|}{ Religión } \\
\hline Católica & 1628 & 81.8 \\
\hline Cristiana & 122 & 6.1 \\
\hline Evangélica & 110 & 5.5 \\
\hline Atea / agnóstica & 109 & 5.5 \\
\hline Otros $^{*}$ & 22 & 1.1 \\
\hline \multicolumn{3}{|l|}{ Migrante ${ }^{\star *}$} \\
\hline No & 1380 & 69.3 \\
\hline Si & 611 & 30.7 \\
\hline
\end{tabular}

* Testigo de Jehová, Adventista, Protestante, Pentecostés, Mormón, Israelita, Budista

** Viene de provincia a la capital

En la tabla 2, observamos las características de la gravidez y paridad de las participantes en el estudio. La mayoría, tenía un GESTA y PARA, mayor a 3 (46,3\%), $719(36,1 \%)$ tuvo un embarazo a término, $1718(86,3 \%)$ afirmó no haber tenido embarazos pre-término, 1459 (73,2\%) nunca tuvo un aborto, el número de hijos vivos fue mayor o igual a 2 en $724(36,4 \%)$ gestantes, $1040(52,2 \%)$ dijo haber tenido por lo menos 1 embarazo y 1347 $(67,6 \%)$ mencionaron haber tenido un parto vaginal.
Tabla 2. Características de la gravidez y paridad de las gestantes

\begin{tabular}{|c|c|c|}
\hline & $\mathbf{N}$ & $\%$ \\
\hline \multicolumn{3}{|l|}{ GESTA } \\
\hline 1 & 460 & 23.1 \\
\hline 2 & 609 & 30.6 \\
\hline$>=3$ & 922 & 46.3 \\
\hline \multicolumn{3}{|c|}{ Embarazo a término } \\
\hline Primigestas & 648 & 32.6 \\
\hline Segundigestas & 719 & 36.1 \\
\hline Multigestas & 624 & 31.3 \\
\hline \multicolumn{3}{|c|}{ Embarazo pre-término } \\
\hline ninguno & 1718 & 86.3 \\
\hline$>\mid=1$ & 273 & 13.7 \\
\hline \multicolumn{3}{|l|}{ Aborto } \\
\hline ninguno & 1459 & 73.2 \\
\hline$>/=1$ & 532 & 26.8 \\
\hline \multicolumn{3}{|l|}{ Hijos vivos } \\
\hline ninguno & 576 & 28.9 \\
\hline 1 & 691 & 34.7 \\
\hline$>/=2$ & 724 & 36.4 \\
\hline \multicolumn{3}{|l|}{ Parto vaginal } \\
\hline ninguno & 951 & 47.8 \\
\hline$>=1$ & 1040 & 52.2 \\
\hline \multicolumn{3}{|l|}{ Parto cesárea } \\
\hline ninguno & 1347 & 67.66 \\
\hline$>/=1$ & 644 & 32.35 \\
\hline
\end{tabular}

La frecuencia de relaciones sexuales, el deseo sexual, el patrón de respuesta y el miedo a ejercer relaciones sexuales (Tabla 3) disminuyó de periodicidad (de diario/interdiario a cada 11 días a más) mientras iba avanzando el embarazo (por trimestres), lo mismo ocurrió con el miedo a tener actos sexuales, que aumentó de 247(12,6\%) a $1302(76,2 \%)$.

Con relación a la respuesta sexual, en su mayoría, refirieron ausencia de orgasmo durante el coito $(42,8 \%)$.

En la Tabla 3, se puede observar que con relación a la preferencia en la posición para el acto sexual hubo un cambio significativo, pasando de "Él sobre ella" durante el primer trimestre: 934(46,9\%) gestantes, a “De lado" en 956(48\%) gestantes durante el tercer trimestre.

La Figura 1, representa todo lo mencionado anteriormente. Identificando una tendencia que sigue la conducta sexual de las gestantes a través de los trimestres del embarazo. 
Tabla 3. Frecuencia de relaciones sexuales, deseo sexual, patrón del ciclo de respuesta sexual, miedo y posiciones sexuales en gestantes de Lima Metropolitana

\begin{tabular}{|c|c|c|c|c|}
\hline & \multicolumn{4}{|c|}{ n (\%) } \\
\hline & $\begin{array}{l}\text { Antes del } \\
\text { embarazo }\end{array}$ & $\begin{array}{l}\text { Primer } \\
\text { trimestre }\end{array}$ & $\begin{array}{l}\text { Segundo } \\
\text { trimestre }\end{array}$ & $\begin{array}{c}\text { Tercer } \\
\text { trimestre }\end{array}$ \\
\hline \multicolumn{5}{|l|}{$\begin{array}{l}\text { Frecuencia de relaciones } \\
\text { sexuales }\end{array}$} \\
\hline Diaria / interdiaria & $896(45.2)$ & $896(45.2)$ & $229(12.8)$ & $118(7.6)$ \\
\hline Cada 3 días & $819(41.3)$ & $819(41.3)$ & $697(38.8)$ & $338(21.8)$ \\
\hline Cada 11 a más días & $271(13.6)$ & $271(13.6)$ & $867(48.4)$ & $1096(70.6)$ \\
\hline \multicolumn{5}{|l|}{ Deseo sexual } \\
\hline Diaria / interdiaria & $905(45.8)$ & $905(45.8)$ & $295(16.4)$ & $154(9.8)$ \\
\hline Cada 3 días & $788(39.9)$ & 788 (39.9) & $681(37.8)$ & 405 (25.6) \\
\hline Cada 11 a más días & $282(14.3)$ & $282(14.3)$ & $825(45.8)$ & $1020(64.6)$ \\
\hline \multicolumn{5}{|l|}{$\begin{array}{l}\text { Patrón del ciclo de } \\
\text { respuesta sexual }\end{array}$} \\
\hline Mono - orgásmica & $947(47.8)$ & $947(47.8)$ & $826(48.2)$ & $647(415)$ \\
\hline Poliorgásmica & $617(31.1)$ & $617(31.1)$ & $344(19.2)$ & $165(10.6)$ \\
\hline Multiorgásmica & $298 \quad(15)$ & $298 \quad(15)$ & $95(5.3)$ & $80(5.1)$ \\
\hline No orgasmo & 121 (6.1) & 121 (6.1) & $489(27.3)$ & $668(42.8)$ \\
\hline \multicolumn{5}{|l|}{$\begin{array}{l}\text { Miedo a tener relaciones } \\
\text { sexuales durante } \\
\text { el embarazo }\end{array}$} \\
\hline Si & 247 (12.6) & 247 (12.6) & $1170(61.8)$ & $1302(76.2)$ \\
\hline No & $1721(87.4)$ & $1721(87.4)$ & 697 (38.2) & 407 (23.8) \\
\hline \multicolumn{5}{|l|}{$\begin{array}{l}\text { Posiciones sexuales } \\
\text { durante el embarazo* }\end{array}$} \\
\hline Ella sobre él & & $771 / 1991 \quad$ (39) & $492 / 1991$ (24.7) & 213/1991 (10.7) \\
\hline El sobre ella & & $934 / 1991$ (46.9) & $533 / 1991$ (26.8) & $185 / 1991 \quad(9.3)$ \\
\hline De lado & & $562 / 1991(28.2)$ & $852 / 1991$ (42.8) & $956 / 1991$ \\
\hline $\begin{array}{l}\text { De rodillas penetración } \\
\text { por detrás } \\
{ }^{*} \text { Frecuencias expresada }\end{array}$ & al(\%) & 272/1991 (13.7) & 224/1991 (11.3) & 296/1991 (14.9) \\
\hline
\end{tabular}

\section{DISCUSIÓN}

La gestación, conlleva a una serie de cambios anatómicos, funcionales y emocionales que pueden alterar su relación marital. Puede experimentar discomfort o dolor durante el coito y seguir consintiendo a su marido, lo que podría condicionar desarmonía entre ellos (15).

La actividad sexual es esencial para la salud física y mental del individuo. Diversos estudios, han demostrado que la misma disminuye a medida que se incrementa la edad gestacional $(6,10,14)$, modificando su conducta $(3-8,12)$. La misma que podría ser condicionada por la conducta del hombre, quién podría tener satisfacción sexual vía extra-marital (16).
Tema que no fue explorado en la presente investigación.

A nuestro conocimiento, este es el estudio más grande y representativo de nuestro medio, para evaluar la actividad sexual de la gestante sin riesgo obstétrico.

Luego de ver los resultados, observamos una reducción del deseo sexual, la frecuencia de las relaciones sexuales y el ciclo de respuesta con el paso de los trimestres.

Sólo en un estudio (5), éstas características se mantuvieron constantes, trabajo que menciona que una de cada cinco gestantes se sentía presionada 

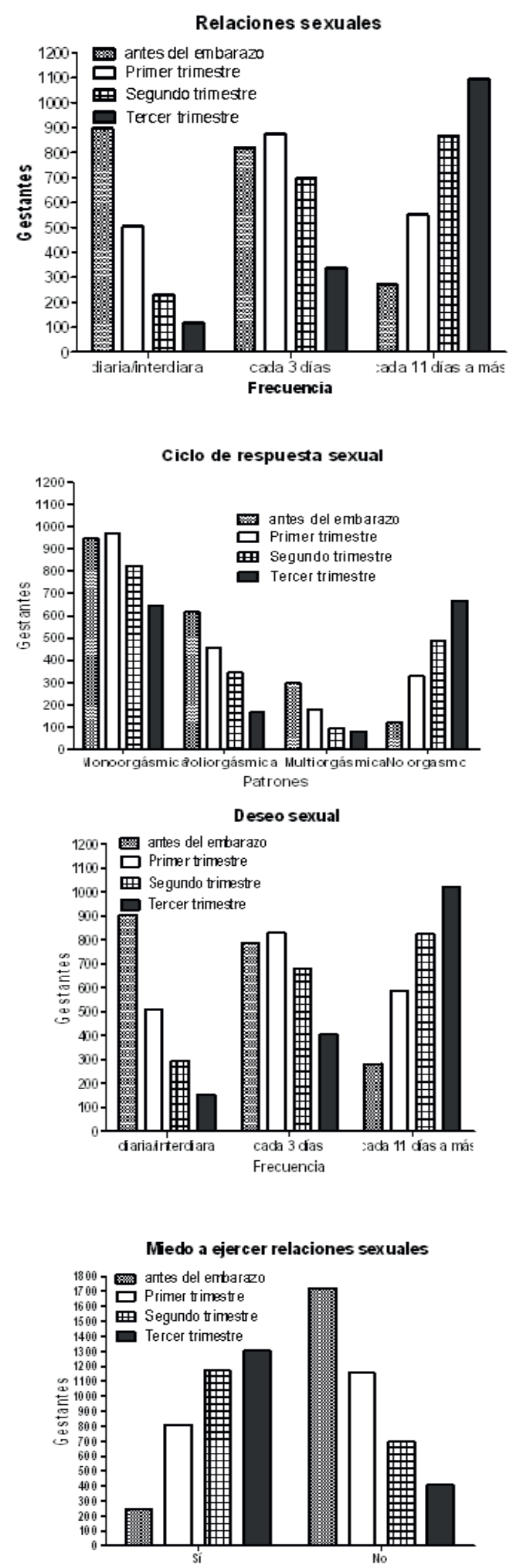

Figura 1. Frecuencia de relaciones sexuales, deseo sexual, ciclo de respuesta sexual y miedo a ejercer relaciones sexuales en gestantes de Lima metropolitana. para tener relaciones sexuales. Exigencia de la pareja que explicaría sus resultados.

Más del 70\% de las encuestadas compartía de manera cotidiana su vida familiar, por lo que la muestra fue representativa para entender que a pesar de verse y tener encuentros sexuales con facilidad, "naturalmente", postergan progresivamente sus encuentros maritales.

Son varios los factores que han sido analizados para justificar la disminución en la actividad sexual durante la gestación: cambio en la imagen corporal, temor a dañar al feto, dispareunia y el consejo médico $(17,18)$. Incluso existe el pensamiento que la actividad sexual puede causar aborto 0 parto pretérmino (19), lo que podría explicar la disminución del coito a medida que se acerca el momento del parto.

Lo que se evidencia en la Figura 1, al observar de manera significativa el incremento del miedo, a medida que se avanza con el embarazo, especialmente en el tercer trimestre.

El orgasmo y placer sexual, también disminuyen a medida que progresa el embarazo. Sin embargo, debemos considerar que incluso desde antes del inicio de la gestación, 121 gestantes refirieron no tener esa experiencia. Por lo que, se hace necesario considerar el asesoramiento preconcepcional para informar y explicar sobre los diferentes aspectos de la sexualidad humana.

Y una vez iniciado el embarazo, durante las sesiones de Psicoprofilaxis Obstétrica, preparar a la pareja en todo lo relacionado a su sexualidad y salud reproductiva (20).

Con relación al cambio de las posiciones sexuales, en nuestro estudio encontramos una tendencia a la práctica de la posición “de lado”. Iguales resultados han sido publicados anteriormente (4), lo que se asociaría a la complacencia mutua y donde se prioriza la comodidad de la mujer.

Otro aspecto a considerar -tema no explorado en esta investigación-, es lo relacionado al tipo de personalidad y la manera como afronte la pareja su 
sexualidad, tema especialmente muy vinculado a la mujer $(21,22)$.

La actividad sexual y la manera como la pareja decida realizarla, siempre será muy conveniente explorar. Hoy, por ej emplo; se conoce que existe una preferencia al rasurado del vello púbico $(23,24)$, por lo que siempre tendremos la oportunidad de seguir investigando acerca de la sexualidad y genitalidad de la población.

Finalmente, es necesario tener en cuenta que el diseño del estudio fue transversal y no prospectivo, lo que hubiese permitido mayor análisis, ya que en este estudio se ve el cambio de las conductas sexuales con el paso de los trimestres.

En conclusión, la actividad sexual en las gestantes de Lima Metropolitana tiende a disminuir en frecuencia, deseo, y ciclo de respuesta.Fue significativo el cambio de posición en el acto sexual, siendo la "de lado" la más utilizada en el tercer trimestre del embarazo.

\section{REFERENCIAS BIBLIOGRÁFICAS}

1. Dunkel Schetter C. Psychological Science on Pregnancy: Stress Processes, Biopsychosocial Models, and Emerging Research Issues. Annu Rev Psychol. 2011;62(1):531-58.

2. J $m$ F. Physiology of the pregnant woman and risk factors. Contracept Fertil Sex. noviembre de 1993;21(11):811-5.

3. Maternal sexuality during pregnancy and after childbirth in muslim Kuwaiti women - Springer [Internet]. [citado 17 de mayo de 2015]. Recuperado a partir de: http: / /link.springer. com/article/10.1007/BF01541581.

4. Tamayo M, Calderón V, Colunga S. Conductas sexuales durante el embarazo. Santiago 2003; 101:213-30.

5. La Rossa R. Sex during Pregnancy: A Symbolic Interactionist Analysis. J Sex Res. 1 de mayo de 1979;15(2):119-28.

6. Bartellas E, Crane JMG, Daley M, Bennett KA, Hutchens D. Sexuality and sexual activity in pregnancy. BJOG Int J Obstet Gynaecol. 1 de agosto de 2000;107(8):964-8.

7. Von Sydow K. Sexuality during pregnancy and after childbirth: A metacontent analysis of 59 studies. J Psychosom Res. julio de 1999;47(1):27-49.
8. Alonso LM, Pérez MA, Arias C, Figueroa N, Gamarra C, Martínez $A$, et al. Características biopsicosociales y frecuencia de relaciones sexuales de las embarazadas en la ESE Prudencio Padilla Clínica Sur. Barranquilla (Colombia). Salud Uninorte. 2004;18(1):8-19.

9. Falicov CJ. Sexual adjustment during first pregnancy and post partum. Am J Obstet Gynecol. 1 de diciembre de 1973;117(7):991-1000.

10. Von Sydow K. Female sexuality and historical time: a comparison of sexual biographies of German women born between 1895 and 1936. Arch Sex Behav. octubre de 1996;25(5):473-93.

11. García LM. Validez y Fiabilidad. [citado 17 de mayo de 2015]; Recuperado a partir de: http://mail.cochrane.es/files/ ValidezFiabilidad_0.pdf

12. Brandão ER, Heilborn ML. Sexualidade e gravidez na adolescência entre jovens de camadas médias do Rio de Janeiro, Brasil Middle-class teenage sexuality and pregnancy in Rio de Janeiro, Brazil. Cad Saúde Pública. 2006;22(7):142130.

13. Guibovich A. Conocimientos sobre educación sexual en madres adolescentes atendidas en el Hospital Nacional Arzobispo Loayza. Horiz Med 2012; 12(4):35-42.

14. Adinma Jl. Sexuality in Nigerian pregnant women: perceptions and practice. Aust N Z J Obstet Gynaecol 1995; 35:290-293.

15. Bello FA, Olayemi O, Aimakhu CO, Adekunle AO.Effect of pregnancy and childbirth on sexuality of women in Ibadan, Nigeria. ISRN Obstet Gynecol 2011;2011:856-86.

16. Onah H, lloabachie G, Obi S, Ezugwu F. Eze J. Nigerian male sexual activity during pregnancy. Int J of Gynecology \& Obstetrics 2002; 76:219-223.

17. Aslan G, Aslan D, Kizilyar A, Ispahi C, Esen A. A prospective analysis of sexual functions during pregnancy. Int J Impot Res 2005; 17(2):154-7.

18. Fox WY, Chan LY, Yuen PM. Sexual behavior and activity in Chinese pregnant women. Acta Obstet Gynecol Scand 2005; 84(10):934-8.

19. Babazadeh R, Mirzaii K, Masomi Z. Changes in sexual desire and activity during pregnancy among women in Shahroud, Iran. Int J of Gynecology and Obstetrics 2013; 120:82-84. 
20. Morales S, Guibovich A, Yabar M. Psicoprofilaxis Obstétrica: Actualización, definiciones y conceptos. Horiz Med 2014; 14(4):53-57.

21. Sloane HM. Tales of a reluctant sex radical: barriers to teaching the importance of pleasure for wellbeing. Sexuality and Disability 2014; 32(4):453'67.

22. Crisp C, Vaccaro C, Fellner A, Kleeman S, Pauls R. The influence of personality and coping on female sexual function: A population Survey. J Sex Med 2015; 12:109-115.

\section{Fuentes de financiamiento}

Este artículo ha sido financiado por los autores.

\section{Conflictos de interés}

Los autores declaran no tener ningún conflicto de interés.
23. Struckman-Johnson C, Gaster S, Struckman-Johnson D. A preliminary study of sexual activity as a distraction for young drivers. Accident Analysis and Prevention 2014; 71:120-128.

24. Butler S, Smith N, Collazo E, Caltabiano L, Herbenick D. Public hair preferences, reasons for removal, and associated genital Symptoms: Comparisons between men and women. J Sex Med 2015; 12:48-58.

\section{Correspondencia:}

Alex Guibovich Mesinas

Dirección: Nicolás Alcázar 730, Pueblo Libre. Lima-Perú.

Teléfono: +51 999653059

Correo: alexguibovichmesinas@yahoo.es 